\title{
Hybrid Colloidal Quantum Dot Silicon Nitride Waveguide Gain Measurement Based on Variable Stripe Length Method
}

\author{
Yunpeng Zhu ${ }^{1,3}$, Weiqiang Xie ${ }^{1,3}$, Pieter Geiregat ${ }^{1,2,3}$, Suzanne Bisschop ${ }^{1,2,3}$, Tangi Aubert ${ }^{2,3}$, Edouard \\ Brainis $^{2,3}$, Zeger Hens ${ }^{2,3}$, Dries van Thourhout ${ }^{1,3}$ \\ ${ }^{1}$ Photonics Research Group, INTEC Department, Ghent University-IMEC, Ghent B-9000, Belgium \\ ${ }^{2}$ Physics and Chemistry of Nanostructures, Ghent University, Krijgslaan 281-S3, 9000 Ghent, Belgium \\ ${ }^{3}$ Center of Nano and Biophotonics (NB Photonics), Ghent University, Ghent B-9000, Belgium \\ Author e-mail address: yunpeng.zhu@ugent.be
}

\begin{abstract}
We fabricated hybrid colloidal quantum dot silicon nitride waveguides and demonstrate they exhibit amplified spontaneous emission under femtosecond optical pumping. The gain coefficient is measured using an integrated variable stripe length method. OCIS codes: (130.0130) Integrated Optics; (140.0140) Laser and Laser Optics
\end{abstract}

\section{Introduction}

Given its relatively high index ( 2.0) and broad optical transparency window, silicon nitride ( $\mathrm{SiN}$ ) is considered a promising material for realizing compact photonic ICs, e.g. for use in bio sensing or nonlinear optics. While high quality passive functionality has been demonstrated for SiN PICs [1], optically active functionality is still largely missing. Colloidal quantum dots (CQDs) on the other hand exhibit unique optical properties. They can emit light with tunable luminescence and high quantum yield. Under femtosecond and nanosecond pumping, they have shown amplified spontaneous emission (ASE) and lasing [2]. Hence combining CQDs with SiN waveguides could provide the basis of a PIC-platform also containing active functionality.

In this work, we embed $\mathrm{CdSe} / \mathrm{CdS}$ core/shell CQDs in SiN stripes, to form an emissive hybrid QD/SiN waveguide. Importantly, the CQDs preserve their original emission properties during the different processing steps. With femtosecond optical pumping, the sandwich type hybrid waveguide shows ASE. The variable stripe length method [3] has been used out to estimate the optical gain coefficient.

\section{Fabrication}

The sandwich type hybrid colloidal quantum dot $\mathrm{SiN}$ waveguide is fabricated as follows. Starting from a silicon wafer with a $3 \mu \mathrm{m}$ thermal oxide on top, first, a $100 \mathrm{~nm}$ thick SiN layer is deposited. The deposition is performed using an optimized plasma enhanced chemical vapor deposition (PECVD) process at a temperature of $270^{\circ} \mathrm{C}$. Then the "flash" synthesized CdSe/CdS core-shell CQDs [4] are spin coated on top of this SiN layer to form a densely packed layer with a thickness of around 70nm. Another $100 \mathrm{~nm}$ thick SiN layer is deposited onto the CQDs layer to form the full sandwich layer stack. The deposition temperature for the top layer is reduced to $120^{\circ} \mathrm{C}$ only, to limit damage to the CQDs. Contact lithography followed by reactive ion etching (RIE) are used to define the waveguides.
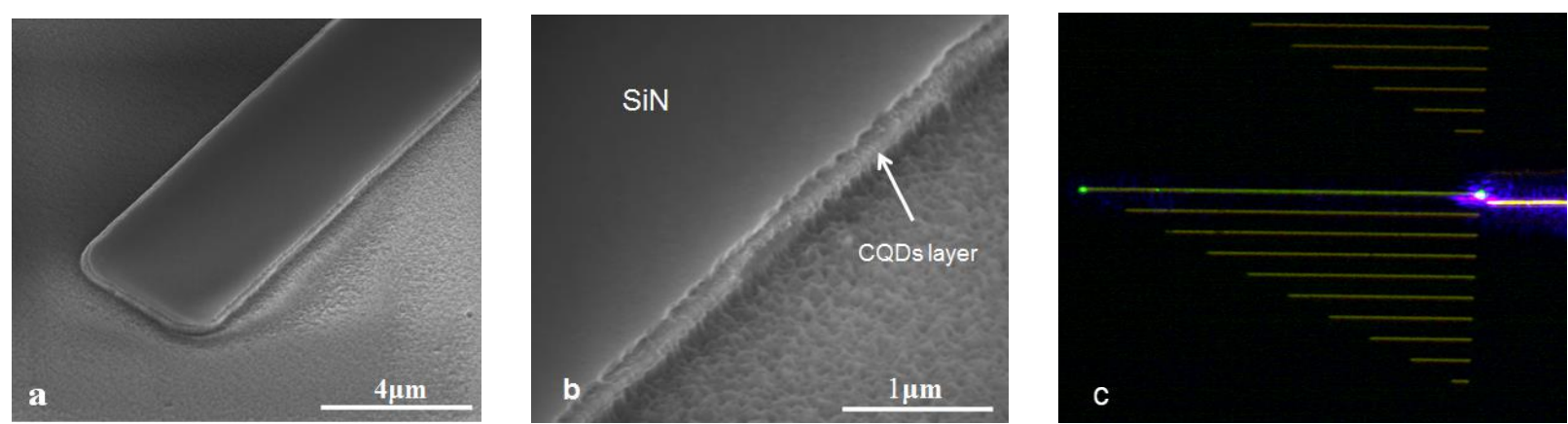

Fig.1. (a) SEM picture of the fabricated hybrid waveguide. (b) The zoom in SEM picture of the fabricated hybrid waveguide sidewall. We can see the embedded CQDs layer has been etched through. (c) Microscope picture of the emitting waveguide and the multimode fiber

All the recipes have been optimized such that low-loss waveguides are obtained [5]. The samples are cleaved before RIE to ensure that the waveguides are terminated with an uniform facet, which is very important for the later 
measurement. Fig.1 (a) shows a scanning electron microscope picture (SEM) of the fabricated device. Fig.1 (b) is the zoomed in picture of the waveguide sidewall.

\section{Characterization and Results}

Characterization of the waveguide emission is performed with a femtosecond laser system. The Spitfire ACE laser can provide $110 \mathrm{fs}$ pulses at $1 \mathrm{kHz}$ repetition rate with up to $4 \mathrm{~mJ}$ per pulse at $800 \mathrm{~nm}$. A Beta Barium Borate (BBO) crystal is used to up convert some of the $800 \mathrm{~nm}$ light to $400 \mathrm{~nm}$, which is then used to pump the embedded CQDs. A cylindrical lens focuses the pumping laser beam into a line shape of $\pm 8 \mathrm{~mm}$ by $75 \mathrm{um}$. The beam is adjusted to overlap with the waveguide and the light emitted from the waveguide facet is collected by a multimode fiber. Fig. 1 (c) shows the microscope picture of the emitting waveguide and the multimode fiber used to capture the emission.
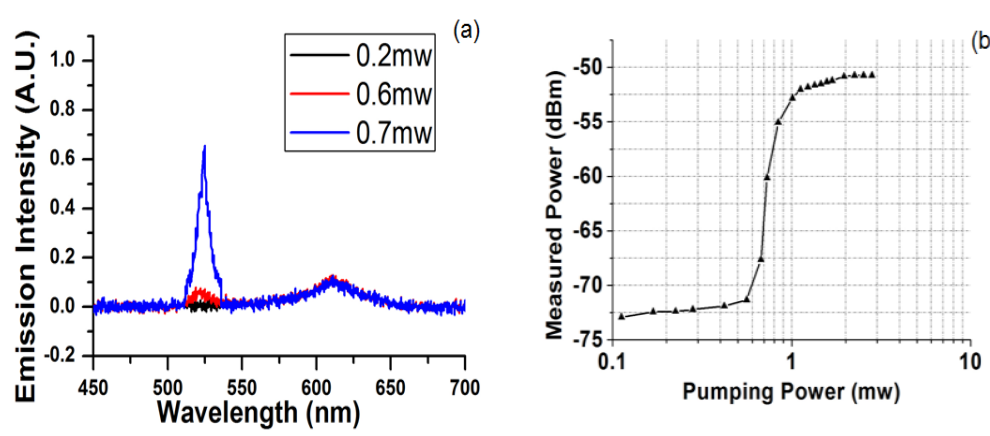

(b)

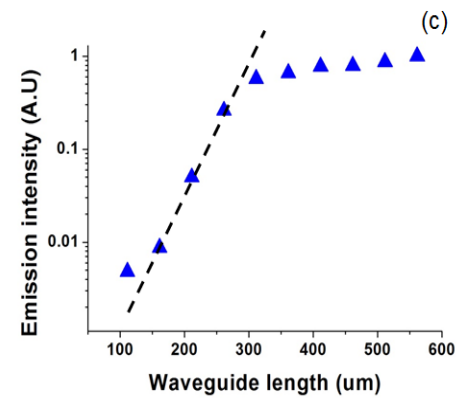

Fig.2. (a) Emission spectrum with different pumping power level; (b) The power dependence measurement : emission from the waveguide with different pumping power level; (c) The measured output power of waveguide with different length

Fig. 2(a) shows the emission spectrum from a $430 \mu \mathrm{m}$ long, $5 \mu \mathrm{m}$ wide waveguide under different pumping levels. Fig. 2(b) shows the output power vs. pumping power for this waveguide. An obvious onset of ASE around $0.6 \mathrm{mw}$ is visible. For higher powers, the $523 \mathrm{~nm}$ ASE peak becomes dominant and green emission from the shell gets amplified.

The traditional variable stripe length method for optical gain measurement usually use a variable slit to control the pumping area. It has some inherent problems, such as light spreading in the layer, position dependent of the out coupling and etc. Our integrated variable stripe length method excludes these problems. To determine the modal gain coefficient of the structure we use a set of waveguides with fixed width $(4 \mu \mathrm{m})$ and variable length, with a fixed pump power of $1 \mathrm{~mW}$. The length difference between adjacent waveguides is 50um. The results are shown in Fig. 2 (c). From a simple fitting in the linear region of the curve a modal gain coefficient of $383 \mathrm{~cm}^{-1}$ is extracted. Measurements at different pump levels will be presented at the conference.

\section{Conclusion}

We fabricated hybrid CQDs SiN waveguides. We demonstrate that CQDs can act as an active material for the SiN platform. The embedded CQDs keep their good optical properties after the full embedding process. SiN provides a good integration platform to help investigating CQDs' optical properties. The hybrid waveguide exhibits ASE under femtosecond laser pumping. The gain coefficient is measured using a variable stripe length method.

This work was partly supported by the EU through the projects ERC-ULPPIC and ICT-NAVOLCHI and by the Belgian Science Policy Office (IAP).

\section{References}

[1] Shah Hosseini, Ehsan, et al. "High quality planar silicon nitride microdisk resonators for integrated photonics in the visible wavelength range." Optics Express 17.17 (2009): 14543-14551.

[2] Klimov, Victor I., et al. "Single-exciton optical gain in semiconductor nanocrystals." Nature 447.7143 (2007): 441-446.

[3] Dal Negro, L., et al. "Applicability conditions and experimental analysis of the variable stripe length method for gain measurements." Optics

Communications 229.1 (2004): 337-348.

[4] Cirillo, Marco, et al. "“Flash" Synthesis of CdSe/CdS Core-Shell Quantum Dots." Chemistry of Materials 26.2 (2014): 1154-1160.

[5] Xie, Weiqiang, et al. "Low-loss silicon nitride waveguide hybridly integrated with colloidal quantum dots." Optics Express 23.9 (2015):

12152-12160. 